\title{
On a New Whirl-forming Species of Streptomyces
}

\author{
by Ryuji ShINobu* and Yoshihiko KaYamura*
}

Received March 13, 1964

A strain of Streptomyces (Label number No. 738) was isolated from the garden soil in Kishiwada City, Osaka Prefecture. Its characteristics were studied from morphological, as well as physiological stand points, and compared with those of the species described in the past.

According to the results obtained, No. 738 was decided to be a new species and the name "Streptomyces kishiwadensis" was given, after the name of Kishiwada City.

\section{Morphology}

1. Macrocolony: Chrysanthemum pattern (Fig. I, 1).

2. Electron microscopical characteristic: Smooth type (Fig. I, 2).

3. Microscopical characteristic: Whirls could be seen on many synthetic media and organic ones. Ammonium Czapek agar, glycerin starch glutamate agar, and glucose asparagine agar were used in parallel for the studies of the aerial mycelia. Among them, ammonium Czapek agar and glycerin starch glutamate agar were more suitable than glucose asparagine agar, because the aerial mycelia on the former two grew adequately enough to stick themselves to the cover glass and enough to be visible through the direct observation method. Next data were written on the mycelia on ammonium Czapek agar.

Aerial mycelium: Long straight or flexuous; cottony, $0.8 \mu$ in width. Many typical whirls (Nitella type whirls); primary and secondary, radial branches were usually many, 6-10, rarely 20-30 (Fig. I, 3 and II). No spiral.

Conidium: Oval to short cylindrical; $0.8 \mu$ in width; $1.0-1.2 \mu$ in length (Fig. I, 2, 3). Substrate mycelium: Monopodial branches; no fragmentation; $0.5-0.6 \mu$ in width.

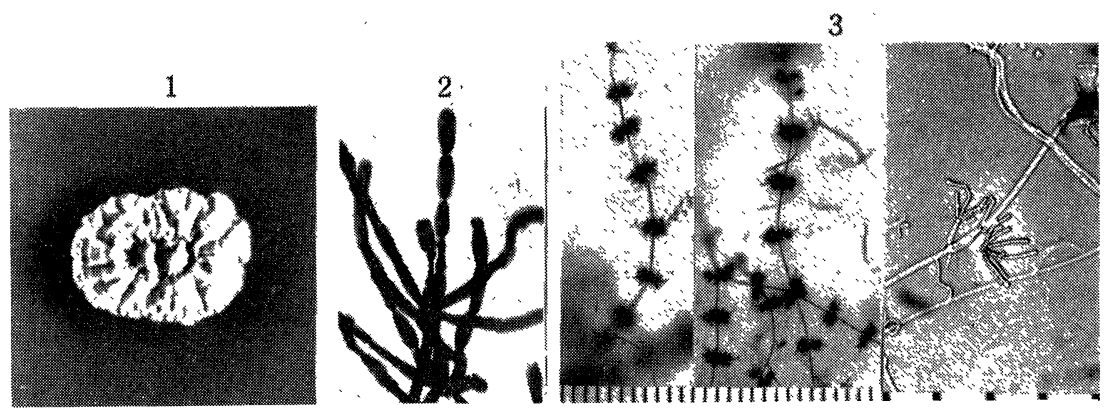

Fig. I. Streptomyces No. 738. 1, colony cultivated on glycerin starch glutamate agar for 20 days; 2, electron-microscopical observation of the conidia, cultivated on ammonium Czapek agar for 10 days; 3, aerial mycelia cultivated on ammonium Czapek agar for 10 days.

* Tennoji Branch, The Osaka University of the Liberal Arts and Education, Osaka, Japan 


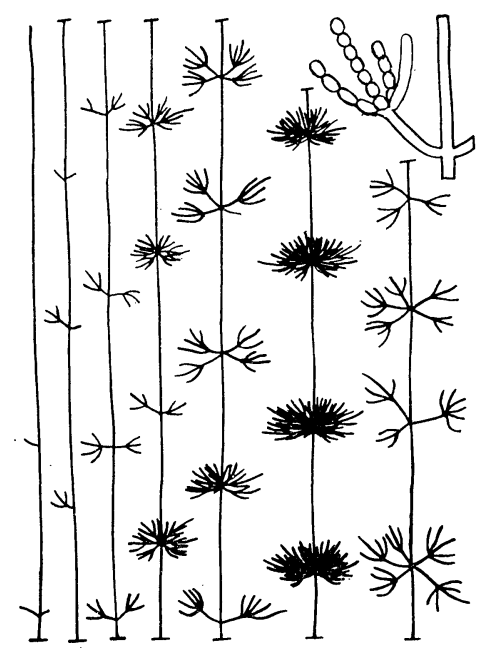

Fig. II. Streptomyces No. 738. Aerial mycelia cultivated on ammonium Czapek agar for 10 days.

\section{Physiological characteristic}

1. Tyrosinase reaction: Positive.

2. Nitrate reduction: Usually negative; sometimes positive, though weak.

3. Diastase reaction by iodine reaction: Clear zones were $6-10 \mathrm{~mm}$ on glycerin starch glutamate agar, incubated for 10 days. No growth on starch agar (Waksman's A).

4. Konjakmannase reaction: Negative.

5. Utilization of carbon sources: Sucrose, trehalose, and inositol were utilized. Xylose, rhamnose, lactose, raffinose, and mannitol were unutilized. Fructose and galactose were uncertain.

\section{Cultural characteristic}

Notes: $\begin{cases}\mathrm{G}: & \text { growth of the colony } \\ \mathrm{A}: & \text { growth of the aerial mycelium and its color } \\ \mathrm{S}: \text {, } & \text { color } \\ \mathrm{P}, 10) & \text { of the substrate mycelium } \\ \mathrm{P}: & \text { production of the soluble pigment }\end{cases}$

1. Ammonium Czapek agar

G: good

A: cottony; good; pale pink-pale brown-pale dull orange

$\mathrm{S}$ : dull yellow orange-maple-pale brown

$\mathrm{P}$ : none

2. Glycerin Czapek agar

G: $\operatorname{good}$

A: good; pale brown-brownish white-pale orange

S: dull yellow-buff

P: buff-pale dull yellow

3. Glucose asparagine agar

G: moderate 
A: moderate-poor; brownish white-pinkish white

S: pale brown-pale dull yellow orange-maple-dull yellow-buff

$\mathrm{P:}$ none

4. Ca-malate agar

G: good

A: partially cottony; good-moderate; brownish white-yellowish; white-pale orange

S : dull yellow orange-buff

$\mathrm{P}$ : none

5. Starch agar (Waksman's A)

G: none-trace

A: none-trace; none-pale pink

$\mathrm{S}$ : none-buff-pale brown

$\mathrm{P}$ : none

6. Urea glycerin agar

G: $\operatorname{good}$

A: good; beige-brownish white

S : buff-dull yellow

P: pale dull yellow-buff

7. Tyrosine agar

G: abundant

A: sometimes some droplets; excellent; blash-beige-pale brown

$\mathrm{S}$ : grayish brown-dark yellowish brown-buff

P: grayish brown-pale buff

8. Glycerin starch glutamate agar

G: good

A: sometimes some droplets; good; pale brown-brownish white-ecru beigebeige-pale pink

S: brown with dark shadow-maple sugar-ochre-brown-dull yellow-buff

P: brown with dark shadow-ochre; sometimes uncertain

Besides the characteristics mentioned above, the following data were obtained.

This strain produced soluble brownish pigment on synthetic agar, bouillon agar, nutrient agar, potato plug, and egg medium, and showed the good growth on organic medium. The color of the aerial mycelia was brownish white-white-pinkish white on synthetic agar; and pale brown-pinkish white-brownish white-light brownish gray, on carrot and potato plugs. Gelatin reaction was unstable and cellulase reaction was negative. Coagulation of milk was partial, and liquefaction was positive.

When these characteristics of this strain were compared carefully in colors of the aerial mycelia and substrate ones with those of Streptomyces described so far, it resembled Str. cinnamomeus f. azacoluta ${ }^{1)}$, Str. reticuli ${ }^{2-6)}$, Str. mashuensis ${ }^{7)}$, and Str. kitasatoensi ${ }^{8}$.

No. 738 produced the soluble pigment on glycerin Czapek agar, Ca-malate agar, urea glycerin agar, tyrosine agar, and glycerin starch glutamate agar, but Str. cinnamomeus f. azacoluta did not do so. Consequently No. 738 was one species and Str. cinnamomeus f. azacoluta another.

Next, the differences between No. 738 and Str. reticuli, Str. mashuensis, and Str. kitasatoensis are shown in the Table 1-3.

Moreover, No. 738 produced the soluble pigments of brown series on most organic 
media, but Str. mashuensis did not.

No. 738 was, therefore, decided to be a new species of Streptomyces and named "Streptomyces kishiwadensis" after the name of Kishiwada City.

Table 1. Differences between No. 738 and Str. reticuli.

\begin{tabular}{|c|c|c|c|}
\hline & & No. 738 & Str. reticuli \\
\hline $\begin{array}{l}\text { Glucose } \\
\text { asparagine } \\
\text { agar }\end{array}$ & $\begin{array}{l}\text { Soluble } \\
\text { pigment }\end{array}$ & none & $\begin{array}{l}\text { brownish, diffusing } \\
\text { through medium }\end{array}$ \\
\hline $\begin{array}{l}\text { Synthetic } \\
\text { agar }\end{array}$ & $\begin{array}{l}\text { Soluble } \\
\text { pigment }\end{array}$ & \multirow{2}{*}{$\begin{array}{l}\text { buff } \\
\text { ambergrow }\end{array}$} & none \\
\hline Egg media & $\begin{array}{l}\text { Soluble } \\
\text { pigment }\end{array}$ & & $\begin{array}{l}\text { purple zone around } \\
\text { growth }\end{array}$ \\
\hline Carrot plug & $\begin{array}{l}\text { Growth } \\
\text { and color } \\
\text { of the aerial } \\
\text { mycelia }\end{array}$ & $\begin{array}{l}\text { good; pale brown } \\
\text { - pinkish white } \\
\text {-brownish white }\end{array}$ & none \\
\hline
\end{tabular}

Table 2. Differences between No. 738 and Str. mashuensis.

\begin{tabular}{|c|c|c|c|}
\hline & & No. 738 & Str. mashuensis \\
\hline \multirow[t]{3}{*}{ Czapek agar } & Growth & good & $\begin{array}{l}\text { yellow colored, reverse of } \\
\text { colony yellow-green colored }\end{array}$ \\
\hline & $\begin{array}{l}\text { Growth } \\
\text { and color } \\
\text { of the aerial } \\
\text { mycelia }\end{array}$ & $\begin{array}{l}\text { good; brownish white } \\
\text {-purplish pink }\end{array}$ & numerous, powdery \\
\hline & $\begin{array}{l}\text { Soluble } \\
\text { pigment }\end{array}$ & buff & none \\
\hline \multirow[t]{2}{*}{$\begin{array}{l}\text { Glucose } \\
\text { asparagine } \\
\text { agar }\end{array}$} & Growth & moderate & $\begin{array}{l}\text { grayish white; powdery, } \\
\text { reverse of colony yel- } \\
\text { lowish-brown }\end{array}$ \\
\hline & $\begin{array}{l}\text { Growth } \\
\text { and color } \\
\text { of the aerial } \\
\text { mycelia }\end{array}$ & $\begin{array}{l}\text { moderate-poor; brownish } \\
\text { white-pinkish white }\end{array}$ & none \\
\hline \multirow[t]{3}{*}{ Starch agar } & Growth & none-trace & $\begin{array}{l}\text { marguerite colony, margin } \\
\text { cottony, primrose pink col- } \\
\text { ored, reverse of colony yel- } \\
\text { low-brown, margin white }\end{array}$ \\
\hline & $\begin{array}{l}\text { Growth } \\
\text { and color } \\
\text { of the aerial } \\
\text { mycelia }\end{array}$ & none-trace; pale pink & $\begin{array}{l}\text { numerous, white cotton } \\
\text { like }\end{array}$ \\
\hline & $\begin{array}{l}\text { Diastase } \\
\text { reaction }\end{array}$ & none & strongly positive \\
\hline
\end{tabular}


Table 3. Differences among No. 738, Str. kitasatoensis (S-7) and Str. kitasatoensis (S-229).

\begin{tabular}{|c|c|c|c|c|}
\hline & & No. 738 & $\begin{array}{l}\text { Str. kitasatoensis } \\
\text { (S-7) }\end{array}$ & $\begin{array}{c}\text { Str. kitasatoensis } \\
\text { (S-229) }\end{array}$ \\
\hline \multirow{3}{*}{$\begin{array}{l}\text { Synthetic } \\
\text { agar }\end{array}$} & Growth & good & poor & poor \\
\hline & $\begin{array}{l}\text { Growth of } \\
\text { the aerial } \\
\text { mycelia }\end{array}$ & good & none & poor \\
\hline & $\begin{array}{l}\text { Color of the } \\
\text { substrate } \\
\text { mycelia }\end{array}$ & $\begin{array}{l}\text { buff--buff with } \\
\text { dark shadow }\end{array}$ & transparent & $?$ \\
\hline \multirow{2}{*}{$\begin{array}{l}\text { Starch } \\
\text { agar }\end{array}$} & Growth & none-trace & moderate & moderate \\
\hline & $\begin{array}{l}\text { Color of } \\
\text { the aerial } \\
\text { mycelia }\end{array}$ & none-pale pink & white, floccose & white, floccose \\
\hline \multirow{3}{*}{$\begin{array}{l}\text { Glucose } \\
\text { asparagine } \\
\text { agar }\end{array}$} & Growth & moderate--poor & none & good \\
\hline & $\begin{array}{l}\text { Color of } \\
\text { the aerial } \\
\text { mycelia }\end{array}$ & $\begin{array}{l}\text { brownish white } \\
\text {-pinkish white }\end{array}$ & \multirow[b]{2}{*}{ pale yellow } & \multirow[t]{2}{*}{ white-pale cream } \\
\hline & $\begin{array}{l}\text { Soluble } \\
\text { pigment }\end{array}$ & none & & \\
\hline
\end{tabular}

\section{References}

1) Pridham, T. G., Shotwell, O. L., Stodola, F. H., Lindenfelser, L. A., Benedict, R. G., and Jackson, R. W., Phytopathology 46: 575 (1956). 2) Breed, R. S., Murray, E. G. D., and Smith, N. R., Bergey's Manual of Determinative Bacteriology Williams \& (Wilkins Co., Baltimore, 6th ed. 1948, 7th ed. 1957). 3) Waksman, S. A., Soil Sci. 8: 71 (1919). 4) , and Lechevalier, H. A., Actinomycetes and their Antibiotics (Williams \& Wilkins Co., Baltimore, 1953). 5) Krassilnikov, N. A., Guide to the identification of bacteria and Actinomycetes (Academy of Science, USSR, Moscow, 1949). 6) Gause, G. F., Preobrazhenskaya, J. P., Kudrina, E. S., Blinov, N. O., Ryabova, I. D., and Sveshnikova, M. A., Problems in the classification of antagonistic Actinomycetes (State Publishing House for Medical Literature, Moscow, 1957). 7) Sawazaki, T., Suzuki, S., Nakamura, G., Kawasaki, Y., and Sekiya, Y., J. Antibiotics 8: 44 (1955). 8) Nakamura, G., Anzai, K., Isono, K., and Suzuki, S., J. Antibiotics, Ser. B 9: 213 (1956). $\quad 9$ ) Shikimei Daijiten (Wada, S. ed., Nippon Shikisai Kenkyusho, Tokyo, 1954). 10) Guide to Color Standard (Wada, S. ed. Nippon Shikisai Kenkyusho, Tokyo, 1951).

\section{摘 要 \\ 信夫隆治・舊村善彦：輸生体を形成する放線菌の一新種について}

大阪府, 岸:和田术の庭園の土堙加ら分離した放線菌 No. 738 は輪生体を形成し, 形態学的, 生理学的 扮上び培養所見的研究加ら, 従来発表された放線菌と異なることを認めたので新種と断定し, 崩和田市の 地名にちなんで Streptomyces kishiwadensis と命名した. (大阪学芸大学天王寺分校) 